\title{
Theoretical Study on Flat Tooth Cone Fracture Type Rock Breaking based on Plane Indentation
}

\author{
Yili Duo ${ }^{1}$, Yujun $\mathrm{Xie}^{2 *}$, and Jun $\mathrm{Hai}^{3}$ \\ ${ }^{1,2,3}$ School of Mechanical Engineering, Liaoning Shihua University, Fushun 113001, \\ China \\ ${ }^{1,2}$ College of Pipeline and Civil Engineering, China University of Petroleum, Qingdao \\ 266580, China \\ 1npuduoyili@163.com, ${ }^{2}$ Inshlnpu@163.com, ${ }^{3}$ Insh_hj@163.com
}

\begin{abstract}
During rock breaking by drill, cone rolls on the rock surface, and contact between gear teeth and the surface is positive Mode-I plane indentation and bias Mode-I plane indentation. During bias action, there will be an asymmetrical Mode-I singular stress field at both ends of the indenter. The stress field singularity is of the same order as the Mode-I crack tip and with the same function distribution. In this paper, based on $J_{2}$ integral conservation law, a new method is established for solving the stress intensity factor of bias Mode-I plane indentation, which not only provides respective stress intensity factor of both ends of indenter but also compares relations between eccentricity, partial load and stress intensity factor. The study not only provides a method for the establishment of a new cone drill engineering design but also improves rock layer boring efficiency.
\end{abstract}

Keywords: Plane indentation, Stress intensity factor, Rock breaking mechanism, Crack

\section{Introduction}

Rock breaking technology is widely used in petroleum, coal mining and other energy industries, underground urban space construction and tunneling shield and other military and civilian facilities, as well as environmental and architectural stone processing [1][2][3]. In 1952, the world's first full face rock tunnel boring machine was developed by the American Robbins Company [4], opening a new era in lithostratigraphy full face boring construction. With the rapid development of the national economy, rock-breaking technology involves numerous engineering such as resource development, transportation, roads, and urban construction. As one of the major basic issues affecting economic and social development, it enjoys particularly prominent importance.

Rock breaking is directly related to driving efficiency and reliability, whose concentrated expression is rock-breaking tool design and related theory. Scholars at home and abroad have undertaken many studies on tool rock breaking mechanism [5][6][7][8][9][10]. Tan Qing studied rock fracture characteristics of boring machine cutterhead under rock temperature variation [11]. The results showed that the higher the rock temperature, the lower rock hardness, mechanical properties and strength. Meanwhile, plus a rapid increase in micro-crack and cracks, rock breaking efficiency is increased. But rock is a poor conductor of heat, so there are certain difficulties in this method to improve rock breaking efficiency. Sung-Oong [12] proposed disc

Article history:

Received (March 2, 2018), Review Result (April 14, 2018), Accepted (May 27, 2018) 
cutter rock breaking numerical research under joint characteristics. The results showed that when disc cutter rock breaking direction was opposite to that of texture, rock breaking input energy would be increased constantly with a decrease in joint angle and spacing. Zhang Zhaohuang, by comparing the mechanical model of interaction between forwarding and positively installed disc cutter and the rock [13][14], proved with plasticity theory that rock breaking specific energy of forwarding installed disk cutter is low. Moreover, the greater radius of the disc cutter, the greater the relative reduction amount of rock breaking specific energy of forwarding installed disc cutter. According to engineering statistics, disc cutter consumption is huge. Frequent replacement is caused due to tool life and fast wear, which not only delays the construction period, causing staff fatigue but also increases project cost. Thus, cone type tool becomes the major rock breaking tool for hard rock heading machine, shield machine and other equipment with wide application.

Stress inducing mode, rock breaking behavior and application of common drill cone, tunneling shield cone hob and hole drilling and other rock breaking tool in rock matrix can be generally divided into Hertz concentrated induction method of spherical indentation stress [15][16], concentrated induction method of cylindrical indentation stress, singular induction method of plane indentation [17][18][19]. Wherein, both Hertz spherical indentation method and plane indentation method have applications in rock breaking engineering. Their common feature is that gear teeth force rock surfaces to induce local stress concentration. Hertz spherical indentation method is mainly characterized by maximum stress in the indenter or central indentation. Despite high peak stress, the radius of the indenter will not be sufficiently small, so peak stress is after all finite. When rock strength is high, the rock-breaking effect is limited, with high energy consumption and large damage area. Rock breaking characteristics of cylindrical gear teeth are similar to that of the Hertz spherical indentation method, which will not be repeated here. However, even when the plane indentation method exerts a little load, high stress will be induced in rock. Hence, the plane indentation method features prominent stress concentration, low load and efficient rock breaking, which enjoys important engineering value. Therefore, in this paper, with a two-dimensional plane indentation for example, through the principle that plane indentation method induces singular stress field on the rock surface, bias indentation stress intensity factor is given based on $\mathbf{J}_{2}$ conservation integration method, eccentricity effect on load size is analyzed, to provide technical support for better results of flat tooth cone drill in rock breaking engineering applications.

\section{Contact pressure problem}

\subsection{Plane Indentation}

In recent years, plane indentation boundary cracking damage has been a major concern. As shown in [Figure 1], plane indentation induced progressive stress field demonstrates $\mathrm{r}^{-1 / 2}$ singularity, with the same order as crack tip singular stress field [20][21][22][23] and essentially the same mechanics. The existence of a singular stress field will inevitably lead to rock damage and cracking. And theoretically, its peak stress can be infinite even under a small load.

It is worth noting that, unlike research objects of conventional fracture mechanics, indentation boundary is general without initial cracks. Under the act of plane indentation singular stress field, cracking problem of indentation boundary is cracking problem without initial crack surface, or crack initiation problem, which carries certain challenges, with difficulty much higher than the cracking problem. However, this kind of indentation-induced singular stress field is near surface stress field, with stress singular point and K control area on 
the surface of the rock mass. The field intensity of the field stress is under the sole control of indentation stress intensity factor Kind. Even if ambient boundary conditions change, singularity and distribution of stress field will not be undermined, with the only impact on the size of indentation stress intensity factor. This characteristic provides an ideal fracture mechanics model for research on flat tooth drilling tool fracture type rock breaking technology and applications, which is especially suitable for the development of new flat tooth cone drilling tool and shield flat hob and can form new and more perfect rock fragmentation theories.

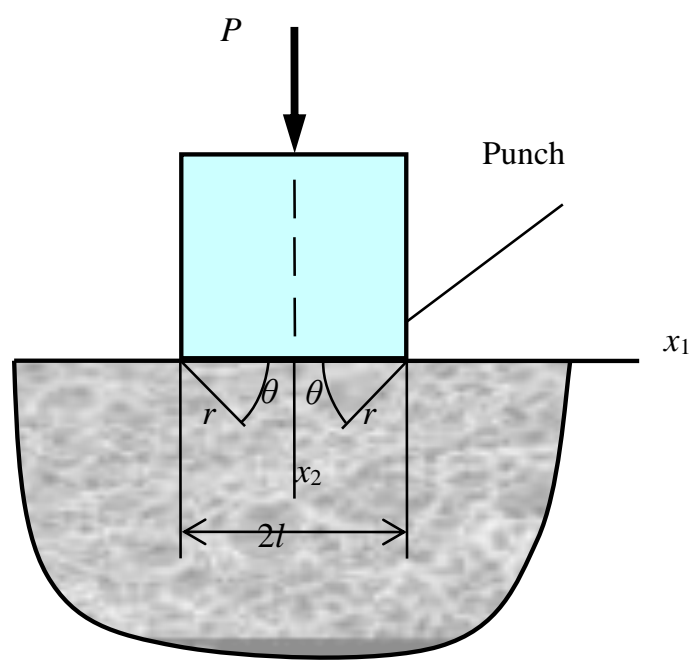

(a) Contact by a rigid flat-end punch

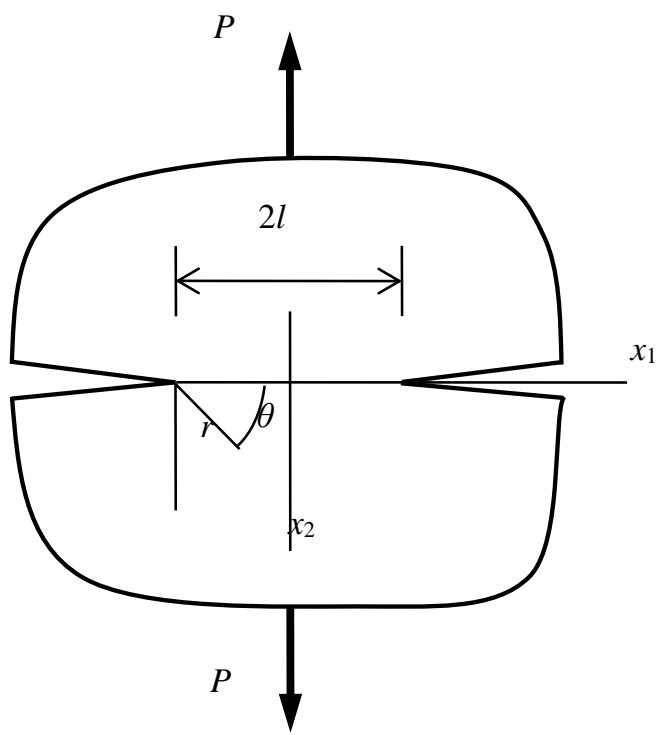

(b) Related Mode I crack

Figure 1. A schematic representation of the contact between a two-dimensional rectangular punch and a substrate

\subsection{Rectangular rigid head contact problem}

Two typical rock-cutting saws commonly used in engineering are shown in [Figure 2]. The common and yet unique character is that these rock-cutting tools can be modeled by periodic rigid and flat tipped indenters. It has been pointed out from the previous work that a singular stress field exists in an incompressible substrate at the sliding contact edge of a rigid flat-ended indenter pressing down onto the substrate.

It should be emphasized that such a singular indentation stress field is sufficient to generate micro-cracks on the contact surface even if it is free of any micro-cracks. This surface cracking mechanism can potentially be utilized to assist rock-cutting, in addition to the diamond grits embedded on the cutter surface.

\section{Plane indentation configuration and singular stress field}

As is shown in [Figure 1 (a)] of the plane indentation contact surface, the width of rectangular rigid indenter is $2 l$, load on rigid indenter is $P$, acting on smooth half-plane, Poisson's ratio is $v$, the elastic modulus is $E$. Indentation interfacial stress distribution according to classical analysis is as follows [24][25]: 


$$
p\left(x_{1}\right)=-\frac{P}{\pi \sqrt{l^{2}-x^{2}}}
$$

When the coordinate transformation is given, make $x=r$ - $l$, expand with binomial distribution method, the pressure distribution between indentation interface near the indenter is as follows:

$$
p\left(x_{1}\right)=-\frac{P}{\pi}\left(\frac{1}{\sqrt{2 l r}}+\cdots\right)
$$

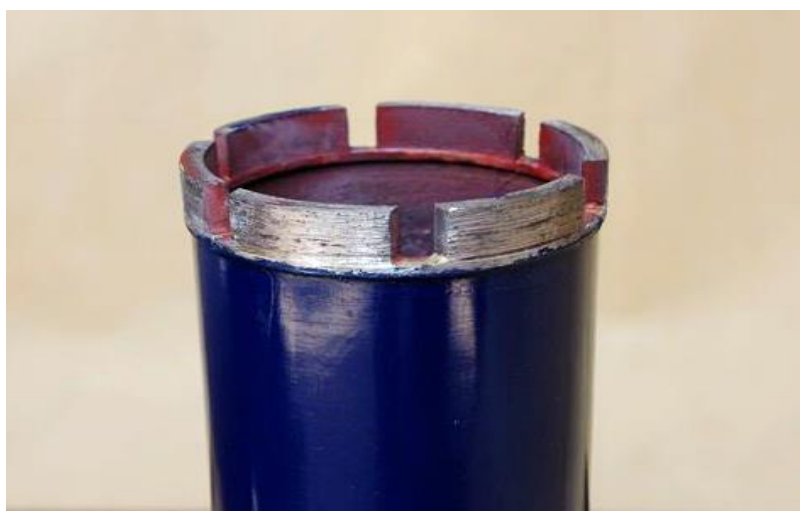

(a)
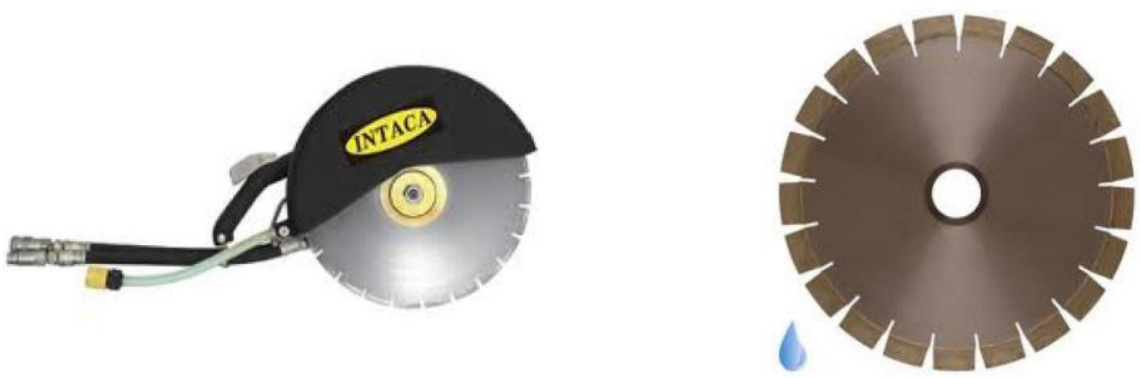

(b)

Figure 2. Two typical rock cutting saws commonly used in engineering

Based on flat end indenter (two-dimensional) induced Mode-I singular stress field, study cracking behavior of indentation boundary. As shown in [Figure 1 (a)], when rigid flat end indenter acts on a half-plane frictionless surface, there is the following stress field in the indentation boundary [26]:

$$
\left(\begin{array}{c}
\sigma_{r r} \\
\sigma_{\theta \theta} \\
\sigma_{r \theta}
\end{array}\right)=-\frac{K_{\mathrm{I}-i n d}}{\sqrt{2 \pi r}}\left(\begin{array}{c}
\cos \frac{\theta}{2}\left(1+\sin ^{2} \frac{\theta}{2}\right) \\
\cos ^{3} \frac{\theta}{2} \\
\sin \frac{\theta}{2} \cos ^{2} \frac{\theta}{2}
\end{array}\right)
$$

Compared with singular stress field Mode-I crack, except negative sign in equation (3), singular stress field in indentation boundary is the same as Mode-I crack problem, 
demonstrating Mode-I singular stress field. Therefore, $K_{\mathrm{I}-\text { ind }}$ is defined as "indentation stress intensity factor" which can be expressed as:

$$
K_{\mathrm{I}-i n d}=\frac{P}{\sqrt{\pi l}}
$$

As can be known from the above equation, for crack shown in [Figure 1 (b)], stress distribution at $x_{2}=0$ is consistent with the indentation problem in [Figure 1 (a)]. Thus, indentation problem in [Figure1 (a)] is equivalent to crack problem in [Figure 1 (b)].

\section{Energy release rate for boundary cracking}

This section describes a method for calculating the energy release rate associated with the initiation of a crack, starting from the free boundary. Consider a three-dimensional elastostatic boundary problem for a material contained within the boundary $S+s$ illustrated in [Figure 3], where the portion s of the boundary is traction-free, and external loading only imposes tractions on $S$. Without changing the boundary conditions on $\mathrm{S}$, impose a continuously varying sequence of static solutions, related to the displacements $u$, given by a time-like parameter " $t$ ". The result of energy release rate per unit time, $\partial \Pi / \partial t$, is given, i.e.,

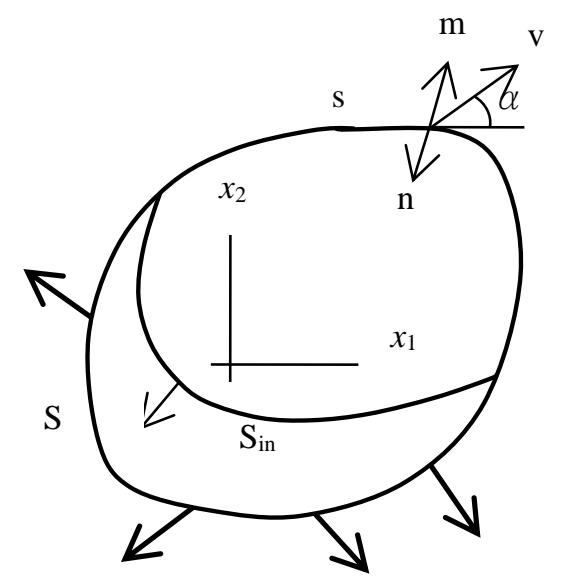

Figure 3. Three-dimensional deformation fields and integration path

$$
\frac{\partial \Pi}{\partial t}=\int_{s} w v_{i} m_{i} d s
$$

where $v_{i}$ denotes the "velocity" of the points on $s$ and $m_{i}$ is the current outward normal to $s$. In the case of two-dimensional deformation fields, relevant to the present problem, the energy release rate remains the same form as Eq.(5).

Let $v_{i}=e_{i}=\triangle_{\mathrm{i}} / \triangle$, which corresponds to two components of unit boundary shift, so that $e_{1}=\cos \alpha, e_{2}=\sin \alpha$, for the two-dimensional solids, whereais the angle between shift $\triangle$ and $e_{1}$ and represents the direction of the boundary shift. Let $n_{i}=-m_{i}$ be the unit inward normal on boundary $s$, which means that the boundary $s$ moves inward, e.g., the case of crack growth. Thus, the energy release rate of boundary shift is given by

$$
\bar{G}=-\frac{\partial \Pi}{\partial \Delta}=\int_{s} w e_{i} n_{i} d s
$$


Now, let all points on the boundary $s$ move in the same direction, and combine this result with the conservation law for two-dimensional problem $\mathrm{J}_{\mathrm{i}}$. The energy release rate expressed by Eq.(6) can then be rearranged along the $v$-direction, i.e.,

$$
\bar{G}=J_{i} e_{i}=J_{1} \cos a+J_{2} \sin a
$$

From the geometrical point of view, boundary cracking or crack initiation regardless of whether it occurs at a crack tip, or a notch, or from a smooth boundary, can be considered as a boundary shift, or a change in the boundary condition, with the limit $s \rightarrow 0$ taken, as shown in [Figure 4]. Then, the energy release rate of boundary cracking can be defined as

$$
G=\left.\left(J_{1}\right)\right|_{s \rightarrow 0} \cos a+\left.\left(J_{2}\right)\right|_{s \rightarrow 0} \sin a
$$

Where $\left.\left(J_{l}\right)\right|_{\mathrm{s} \rightarrow 0}$ denotes the energy release rate of the boundary $s$ shifting in $x_{l}$-direction or the driving force of boundary cracking in $x_{1}$-direction; and $\left.\left(J_{2}\right)\right|_{s \rightarrow 0}$ denotes the energy release rate of the boundary $s$ shifting in $x_{2}$ direction or the driving force of boundary cracking in $x_{2}$ direction when the limits have taken exist.

The geometrical model for crack-tip shattering initiated from a free boundary in $n$ directions with $s=s_{1}+s_{2}+\ldots \ldots+s_{n} \rightarrow 0$ is illustrated in Figure 5 for the case of $\mathrm{n}=3$ and $\mathrm{s}=\mathrm{s}_{1}+\mathrm{s}_{2}+\mathrm{s}_{3} \rightarrow 0$. By following the same procedure from Eqs. (5)-(8), the energy release rate for crack-tip shattering is obtained, i.e.,

$$
G=\sum_{l=1}^{n}\left\{\left.\left(J_{1}\right)\right|_{s_{l} \rightarrow 0} \cos a_{l}+\left.\left(J_{2}\right)\right|_{s_{l} \rightarrow 0} \sin a_{l}\right\}
$$

As expected, the boundary shift or cracking should be moving inwards within the solid, and $\left(\mathrm{J}_{\mathrm{j}}\right)_{\mathrm{s} \rightarrow \rightarrow 0}$ must be positive indicating an energy release.

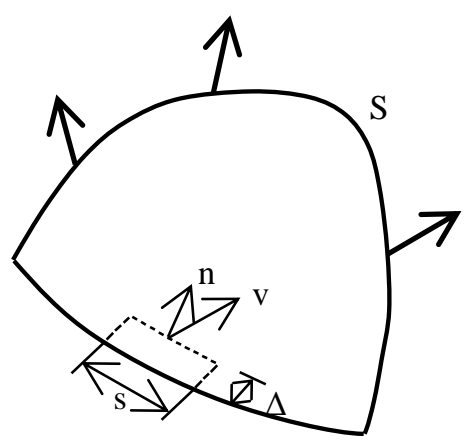

(a) Notch model for boundary cracking

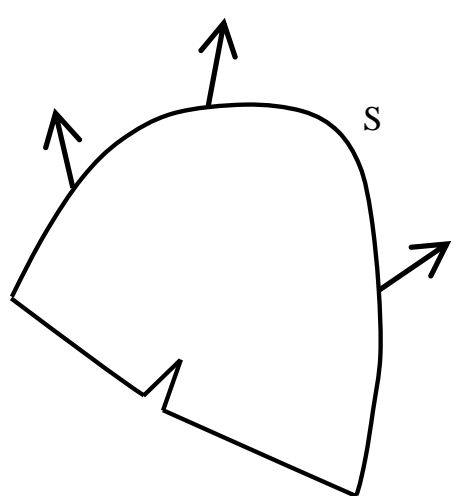

(b) Boundary cracking

Figure 4. Model of boundary cracking when $s \rightarrow 0$ for two-dimensional solids

\section{Conservation law}

\subsection{J Integral}

For a closed curve free of cracks or holes, as shown in Figure 6, based on $\mathrm{J}$ integral conservation law, the following zero integral expression can be obtained [27-28]: 


$$
J_{j}=\int_{s}\left(w n_{j}-T_{i} u_{i, j}\right) d s \quad j=1,2
$$

Eq. (10) contains two integral components $\left(J_{1}\right.$ and $\left.J_{2}\right)$. When the crack plane is parallel to axis $x_{1}, J_{l}$ means crack growth energy release rate, $J_{2}$ means crack opening energy release rate. Both can be used to calculate the stress intensity factor of elastic body cracks, including the calculation of the plane indentation stress intensity factor. This paper will provide the main calculation process for calculating bias Mode-I plane indentation stress intensity factor based on integral $J_{2}$, and will compare eccentricity size.

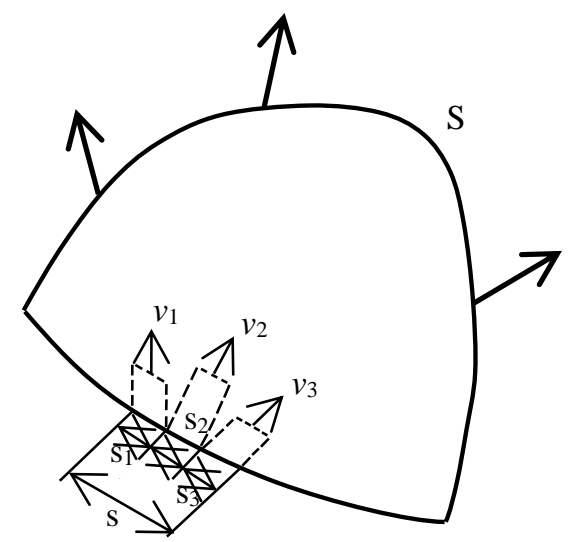

(a) Multi-notch model for boundary scattered-cracking

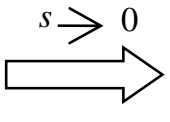

Figure 5. Model of boundary scattered-cracking when $s \rightarrow 0$ for two-dimensional solids

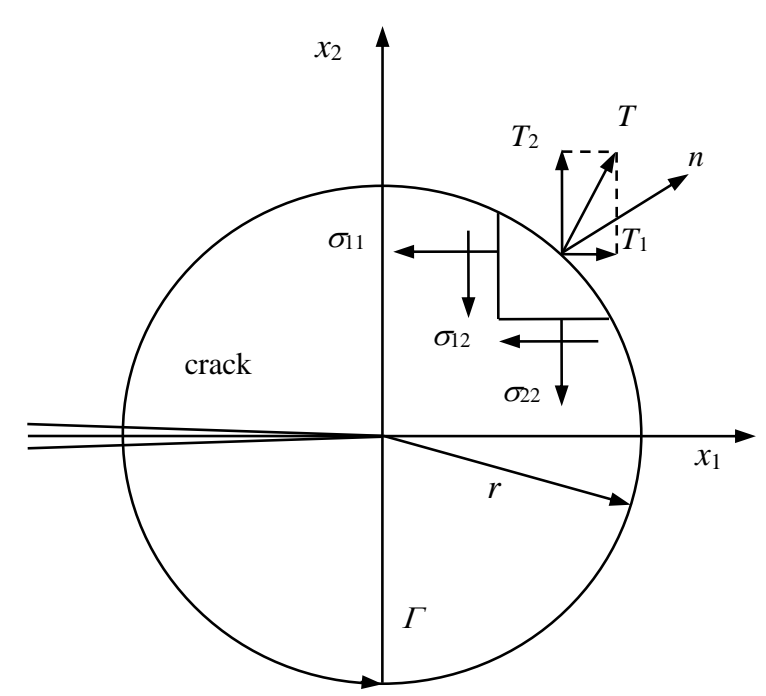

Figure 6. Circular integral path graph

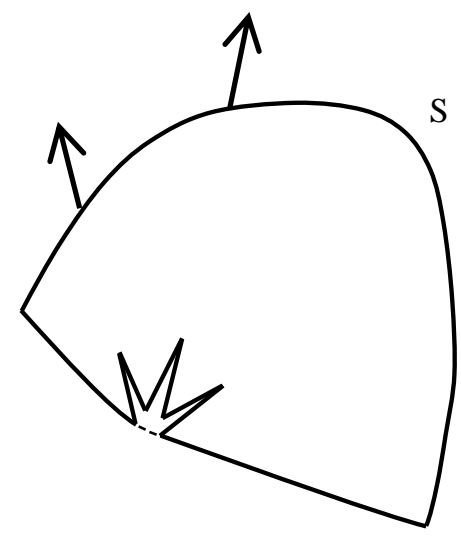

(b) Boundary scattered-cracking

\section{2. $\mathbf{J}_{1}$ and $\mathbf{J}_{2}$ integral calculation of Mode-I crack}

The integral path is a circle with a radius of $r$, the integral is shown in [Figure 6]. Mode-I crack stress field expression is: 


$$
\left\{\begin{array}{c}
\sigma_{11}=\frac{K_{\mathrm{I}}}{\sqrt{2 \pi r}} \cos \frac{\theta}{2}\left(1-\sin \frac{\theta}{2} \sin \frac{3 \theta}{2}\right) \\
\sigma_{22}=\frac{K_{\mathrm{I}}}{\sqrt{2 \pi r}} \cos \frac{\theta}{2}\left(1+\sin \frac{\theta}{2} \sin \frac{3 \theta}{2}\right) \\
\sigma_{12}=\frac{K_{\mathrm{I}}}{\sqrt{2 \pi r}} \cos \frac{\theta}{2} \sin \frac{\theta}{2} \cos \frac{3 \theta}{2}
\end{array}\right.
$$

For homogeneous, isotropia material elastomers, the elastic strain energy density:

$$
w=\frac{1}{2} \sigma_{i j} \varepsilon_{i j}
$$

In Eq. (11) $\theta$ is the angle with the $x_{l}$ axis, rad. Then, substituting Eq. (11) to Eq.(12), we find

$$
w=\frac{(1+v) K_{\mathrm{I}}^{2}}{4 E \pi r}\left[\frac{1}{4}(5-\cos 2 \theta+4 \cos \theta)-2 \mu(1+\cos \theta)\right]
$$

where $v$ is Poisson's ratio, $E$ is Modulus of elasticity, GPA. The expression of surface stress $\mathrm{T}$ is;

$$
\begin{aligned}
& T_{1}=\sigma_{11} n_{1}+\sigma_{12} n_{2} \\
T_{2}= & \sigma_{21} n_{1}+\sigma_{22} n_{2}
\end{aligned}
$$

Substituting Eq. (11) to Eqs. (14) and (15), and the following result can be found

$$
\begin{gathered}
T_{1}=\frac{K_{\mathrm{I}}}{\sqrt{2 \pi r}}\left(\frac{3}{4} \cos \frac{3 \theta}{2}+\frac{1}{4} \cos \frac{\theta}{2}\right) \\
T_{2}=\frac{K_{\mathrm{I}}}{\sqrt{2 \pi r}} \frac{3}{2} \cos \frac{\theta}{2} \sin \theta
\end{gathered}
$$

The expression of Mode-I crack tip displacement field is:

$$
\left\{\begin{array}{l}
u_{1}=\frac{2(1+v) K_{\mathrm{I}}}{4 E} \sqrt{\frac{r}{2 \pi}}\left[(5-8 v) \cos \frac{\theta}{2}-\cos \frac{3 \theta}{2}\right] \\
u_{2}=\frac{2(1+v) K_{\mathrm{I}}}{4 E} \sqrt{\frac{r}{2 \pi}}\left[(7-8 v) \sin \frac{\theta}{2}-\sin \frac{3 \theta}{2}\right]
\end{array}\right.
$$

However, there is a relation between the Cartesian and polar coordinates:

$$
\begin{gathered}
\frac{\partial}{\partial x_{1}}=\cos \theta \frac{\partial}{\partial r}-\frac{\sin \theta}{r} \frac{\partial}{\partial \theta} \\
\frac{\partial}{\partial x_{2}}=\sin \theta \frac{\partial}{\partial r}+\frac{\cos \theta}{r} \frac{\partial}{\partial \theta}
\end{gathered}
$$


Next, we substituting Eq. (18) to Eqs. (19) and (20), can be found as follows:

$$
\begin{gathered}
\frac{\partial u_{1}}{\partial x_{1}}=\frac{(1+v) K_{\mathrm{I}}}{4 E \sqrt{2 \pi r}}\left[(3-8 v) \cos \frac{\theta}{2}+\cos \frac{5 \theta}{2}\right] \\
\frac{\partial u_{2}}{\partial x_{1}}=\frac{(1+v) K_{\mathrm{I}}}{4 E \sqrt{2 \pi r}}\left[(8 v-9) \sin \frac{\theta}{2}+\sin \frac{5 \theta}{2}\right] \\
\frac{\partial u_{1}}{\partial x_{2}}=\frac{(1+v) K_{\mathrm{I}}}{4 E \sqrt{2 \pi r}}\left[(7-8 v) \sin \frac{\theta}{2}+\sin \frac{5 \theta}{2}\right] \\
\frac{\partial u_{2}}{\partial x_{2}}=\frac{(1+v) K_{\mathrm{I}}}{4 E \sqrt{2 \pi r}}\left[(5-8 v) \cos \frac{\theta}{2}-\cos \frac{5 \theta}{2}\right]
\end{gathered}
$$

From Eqs. (16)-(17) and Eqs (21)-(24), will yield

$$
\begin{gathered}
T_{1} \frac{\partial u_{1}}{\partial x_{1}}+T_{2} \frac{\partial u_{2}}{\partial x_{1}}=\frac{(1+v) K_{\mathrm{I}}^{2}}{64 \pi r E}[-2 \cos 3 \theta+(40-48 v) \cos 2 \theta+(18-32 v) \cos \theta+(16 v-24)] \\
T_{1} \frac{\partial u_{1}}{\partial x_{2}}+T_{2} \frac{\partial u_{2}}{\partial x_{2}}=\frac{(1+v) K_{\mathrm{I}}^{2}}{64 \pi r E}[-2 \sin 3 \theta+(40-48 v) \sin 2 \theta+(22-32 v) \sin \theta]
\end{gathered}
$$

From Eqs. (13), (25) and (26), according to Eq. (10), $\mathrm{J}_{1}$ and $\mathrm{J}$ can be expressed as follows:

$$
\begin{aligned}
& J_{1}=\frac{\left(1-v^{2}\right) K_{I}^{2}}{2 \pi E} \int(1-\cos 2 \theta) d \theta \\
& J_{2}=\frac{\left(1-v^{2}\right) K_{I}^{2}}{2 \pi E} \int(-\sin 2 \theta) d \theta
\end{aligned}
$$

\section{Influencing Factors of Flat Tooth Cone Bias}

\subsection{Bias Indentation Stress Intensity Factor}

The positive stress intensity factor problem of the ideally smooth surface has been discussed in the literature [29]. Bias Mode-I plane indentation is shown in [Figure 7], unbalance loading indentation stress intensity factor can be expressed as the superposition of positive $P$ and bending moment $M$ stress intensity factor.

In this paper, finite width rigid indenter with width at $\mathrm{W}$ and thickness at $\mathrm{B}$ (wherein $\mathrm{B}=1$ ) is taken as an example to study stress intensity factor under bias, as shown in Figure 8. In the case of concentrated force, choose a closed path of integration $S_{\text {obcdefghijo }}$ as shown in Figure 8 (a). And the following results can be obtained according to Eq. (10), based on $\mathbf{J}_{2}$ [30][31][32]: 


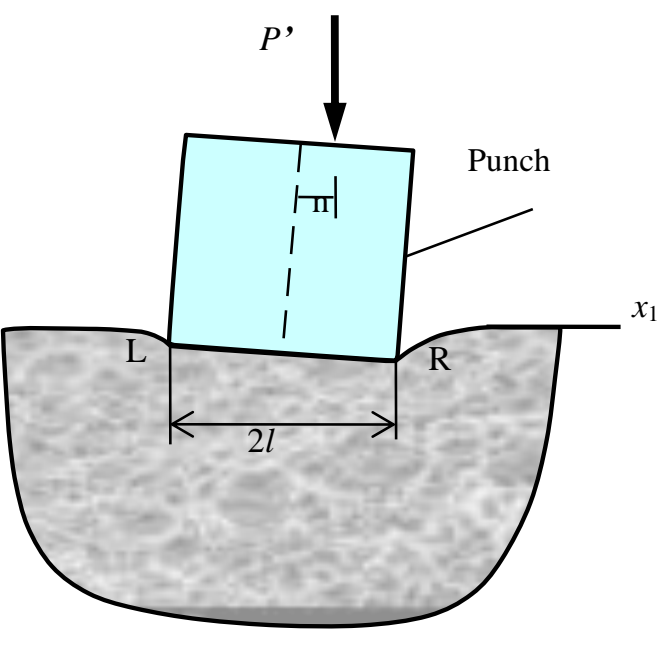

(a)

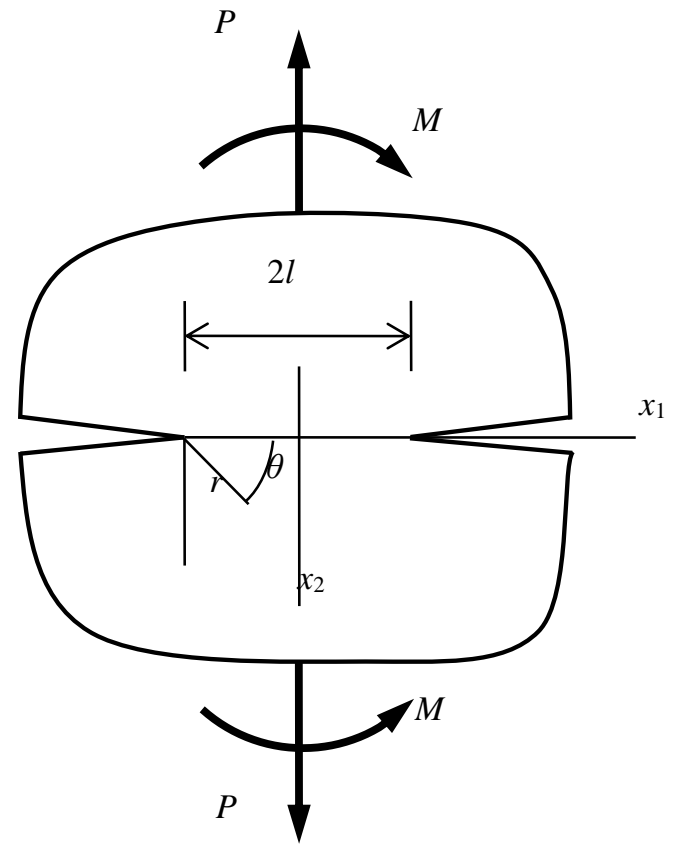

(b)

Figure 7. Bias Mode-I plane indentation

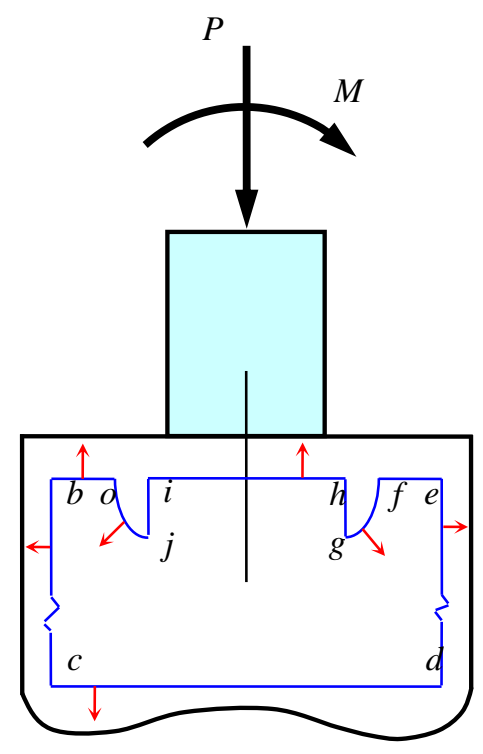

(a)
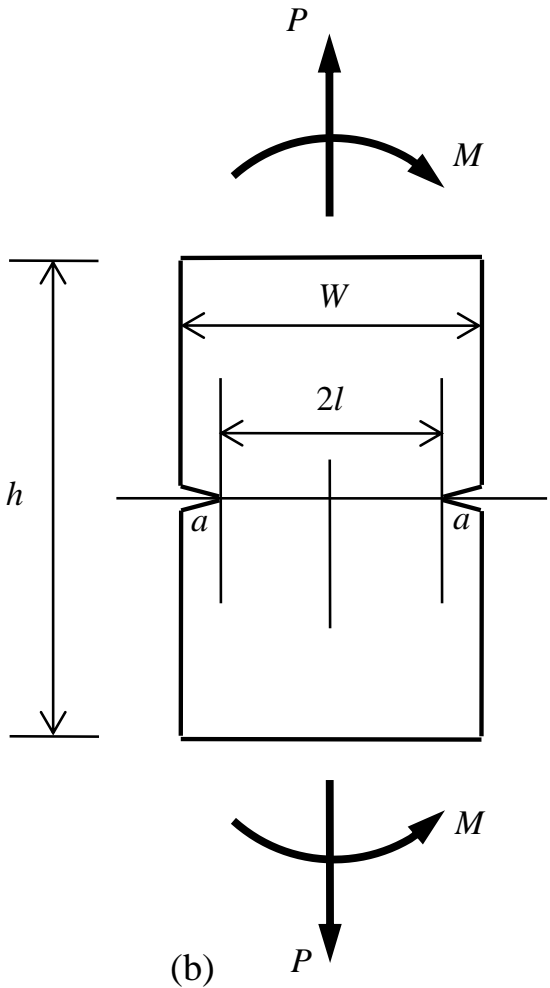

Figure 8. Contact model for elastic solids with finite boundaries and crack model

$$
J_{2-P}=\oint_{S_{\text {obcdefghip }}}\left(w n_{2}-T_{i} u_{i, 2}\right) d s=0
$$


On path $S_{b c}$ and $S_{d e}$, there is $T_{i}=0$ and $n_{2}=0$. And the following calculation results can be obtained:

$$
J_{2-P}=\int_{S_{b c}}\left(w n_{2}-T_{i} u_{i, 2}\right) d s=\int_{S_{d e}}\left(w n_{2}-T_{i} u_{i, 2}\right) d s=0
$$

Path $S_{g h}$ and $S_{i j}$ are straight lines, while path $S_{f g}$ and $S_{j o}$ are quadrants. When $S_{i j o}$ and $S_{f g h}$ are in $K$ - control area at the corner of the indenter, it is not difficult to obtain the following calculation results:

$$
\begin{gathered}
J_{2-P}=\int_{S_{g h}}\left(w n_{2}-T_{i} u_{i, 2}\right) d s=\int_{S_{i j}}\left(w n_{2}-T_{i} u_{i, 2}\right) d s=0 \\
J_{2-P}=\int_{S_{f b}}\left(w n_{2}-T_{i} u_{i, 2}\right) d s=\int_{S_{j o}}\left(w n_{2}-T_{i} u_{i, 2}\right) d s=\frac{\left(1-v^{2}\right) K_{\mathrm{I}-i n d}^{2}}{2 \pi E}
\end{gathered}
$$

For relatively far away indentation contact area and indentation contact area near surface, the following approximate expression can be obtained:

$$
\begin{gathered}
J_{2-P}=\int_{S_{c d}}\left(w n_{2}-T_{i} u_{i, 2}\right) d s=\bar{w}^{-}+P \tilde{u}_{2,2}^{-} \\
J_{2-P}=\int_{S_{h i}}\left(w n_{2}-T_{i} u_{i, 2}\right) d s=-\left(\bar{w}^{+}+P \tilde{u}_{2,2}^{+}\right)
\end{gathered}
$$

Wherein $\tilde{u}_{i}$ represents a strain of neutral axis, which can be obtained by bending theory in material mechanics. The symbol "-" indicates far field cross-section, symbol "+" indicates near field cross-section. $\bar{w}$ represents strain energy density per unit length. Then, substitute equations (30) - (34) into equation (29), and obtain that:

$$
J_{2-P}=\frac{\left(1-v^{2}\right) K_{\mathrm{I}-i n d-P}^{2}}{\pi E}+2 \int_{S_{o b}} w_{P} d s=\left(\bar{w}^{-}+P \tilde{u}_{2,2}^{-}\right)-\left(\bar{w}^{+}+P \tilde{u}_{2,2}^{+}\right)=\frac{P}{2}\left(\tilde{u}_{2,2}^{-}-\tilde{u}_{2,2}^{+}\right)
$$

Wherein, $\tilde{u}_{2,2}^{-}$and $\tilde{u}_{2,2}^{+}$may be expressed as follows [33, 34]:

$$
\begin{gathered}
\tilde{u}_{2,2}^{-}=-\frac{P}{E A} \\
\tilde{u}_{2,2}^{+}=-\frac{P}{E A} \int_{0}^{1} \frac{d \xi}{1-(2 a / W) \sqrt{1-\xi^{2}}}
\end{gathered}
$$

In the formula, $a$ represents crack length, $A=W B$ shows cross-sectional area at the far field $S_{c d}$. Due to movement and cracking of indentation border, and that $S_{o b}$ and $S_{e f}$ are not in the $K$ control area, its integral value is very small which can be ignored. Then substitute Eqs. (36) and (37) into Eq. (35) and obtain:

$$
J_{2-P}=\frac{\left(1-v^{2}\right) K_{\mathrm{I}-i n d-P}^{2}}{\pi E}=\frac{P}{2}\left(\tilde{u}_{2,2}^{-}-\tilde{u}_{2,2}^{+}\right)=\frac{P}{2}\left(\frac{P}{E W} \int_{0}^{1} \frac{d \xi}{1-(2 a / W) \sqrt{1-\xi^{2}}}-\frac{P}{E W}\right)
$$

According to the above formula, stress intensity factor under concentrated force can be expressed as: 


$$
K_{\mathrm{I}-i n d-P}=\frac{\sqrt{\pi} P}{\sqrt{2 W\left(1-v^{2}\right)}}\left(\int_{0}^{1} \frac{d \xi}{1-(2 a / W) \sqrt{1-\xi^{2}}}-1\right)^{1 / 2}
$$

Under concentrated moment, closed integration path $S_{\text {obcdefghijo }}$ is still chosen. As shown in [Figure 8 (a)], it can be obtained according to integral conservation law and by referring to the above calculation method that:

$$
J_{2-M}=\frac{\left(1-v^{2}\right) K_{\mathrm{I}-i n d-M}^{2}}{\pi E}+2 \int_{S_{a b}} w_{M} d s=\left(\bar{w}^{-}-M^{-} \tilde{\varphi}^{-}\right)-\left(\bar{w}^{+}-M^{+} \tilde{\varphi}^{+}\right)=\frac{M}{2}\left(\tilde{\varphi}^{+}-\tilde{\varphi}^{-}\right)
$$

In the formula, $\tilde{\varphi}^{+}$and $\tilde{\varphi}^{-}$are curvature of cracked cross-section and crack-free crosssection after deformation [35]. I represent the inertia moment of the neutral axis of the crosssection (wherein, $B=1$ ).

$$
\begin{gathered}
\tilde{\varphi}^{-}=\frac{M}{E I}, I=\frac{B W^{3}}{12} \\
\tilde{\varphi}^{+}=\frac{M}{E I} \int_{0}^{1} \frac{d \xi}{\left.(1-2 a / W) \sqrt{1-\xi^{2}}\right)^{3}}
\end{gathered}
$$

Then, substitute equation (41) and (42) into equation (40), $M=n P$ [Figure 8 (a)] and stress intensity factor under concentrated moment can be obtained after calculation:

$$
K_{I-i n d-M}=\frac{P \sqrt{\pi}}{\sqrt{2 W\left(1-v^{2}\right)}}\left(\int_{0}^{1} \frac{12 \times\left(\frac{n}{W}\right)^{2}}{\left(1-2 a / W \sqrt{1-\xi^{2}}\right)^{3}} d \xi-12 \times\left(\frac{n}{W}\right)^{2}\right)^{1 / 2}
$$

Due to two non-symmetrical Mode-I singular stress field at the indenter corner under eccentric load, as show in [Figure 9], two different stress intensity factors, in this paper, singular stress field at both ends of indenter is defined as L-end Mode-I singular stress field and R-end Mode-I singular stress field. Stress intensity factor at both ends of indenter obtained via Eq. (39) and Eq. (43) is:

$$
\begin{gathered}
K_{\mathrm{I}-i n d-R}=\frac{P \sqrt{\pi}}{\sqrt{2 W\left(1-v^{2}\right)}}\left[\int_{0}^{1} \frac{1}{\left(1-2 a / W \sqrt{1-\xi^{2}}\right.}+\frac{12 \times\left(\frac{n}{W}\right)^{2}}{\left(1-2 a / W \sqrt{1-\xi^{2}}\right)^{3}} d \xi-1-12 \times\left(\frac{n}{W}\right)^{2}\right]^{1 / 2} \\
K_{\mathrm{I}-i n d-L}=\frac{P \sqrt{\pi}}{\sqrt{2 W\left(1-v^{2}\right)}}\left[\int_{0}^{1} \frac{1}{\left(1-2 a / W \sqrt{1-\xi^{2}}\right.}-\frac{12 \times\left(\frac{n}{W}\right)^{2}}{\left(1-2 a / W \sqrt{1-\xi^{2}}\right)^{3}} d \xi-1+12 \times\left(\frac{n}{W}\right)^{2}\right]^{1 / 2}
\end{gathered}
$$

As can be known from Eqs. (44) and (45), this method innovatively provides different stress intensity factors at both ends of the indenter under bias, and takes into account the impact of eccentricity on stress intensity factor. 


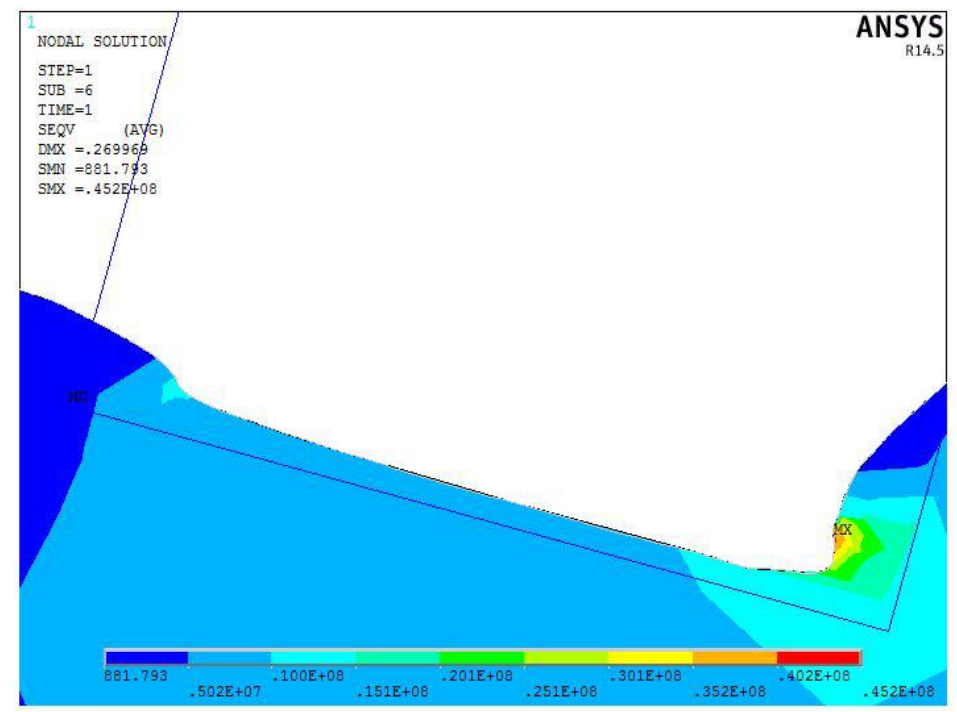

Figure 9. Non-symmetrical Mode-I singular stress field at indenter corner under eccentric load

\subsection{Impact Analysis of Eccentricity}

In this paper, through equations (44) and (45), the effect of different eccentricity values on eccentric load is analyzed. [Figure 10 (a)] shows that in the case of increasing crack expansion, with an increase in eccentricity, the eccentric load will gradually become smaller under constant stress intensity factor; stress intensity factor will gradually become large under constant load. The difference is that, although [Figure 10 (b)] shows that when eccentricity changes from 0.1 to 0.3 , the situation is opposite to the case of [Figure 10 (a)], value change from 0.1 to 0.3 causes a little effect which can be ignored. At the same time, research shows that when eccentricity is> 0.3 , an imaginary number will appear in the stress intensity factor of the left indenter, and with indefinitely growing eccentricity, the left indenter will leave the base. This is not conducive to improving drilling efficiency, which will wear the right indenter and reduce the service life of the indenter. This finding not only enlightens our drilling efficiency improvement but also means great significance for the dimensional design of drill and solution of engineering application problem.

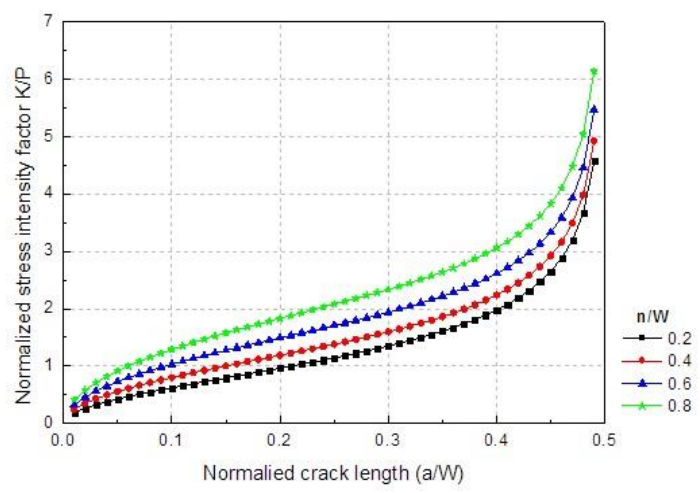

(a) R-end of indenter

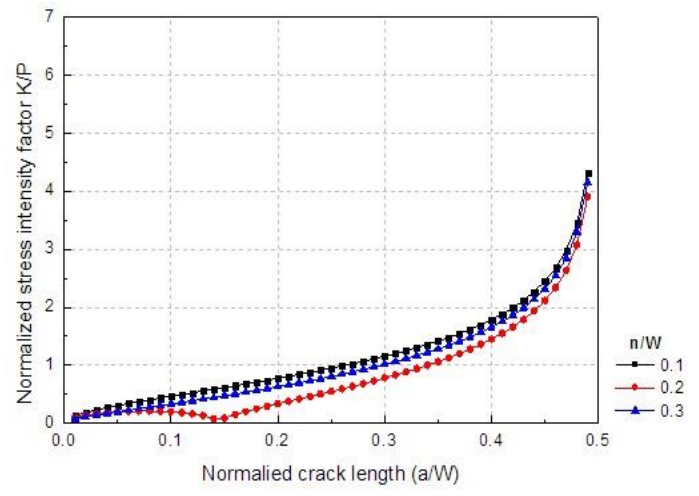

(b) L-end of indenter

Figure 10. Effect of eccentricity on K/P 


\section{Conclusion}

(1) Different stress intensity factors at both ends of the indenter under bias are given based on $\mathbf{J}_{2}$ integral conservation law, and the calculation process is simple with good accuracy, which can provide a reference for engineering application.

(2) When the stress intensity factor remains constant, eccentricity growth can reduce input load, but when eccentricity is $\geq 0.3$, the imaginary number will appear in the stress intensity factor of the left indenter; when the stress intensity factor is constant, indenter width reduction can also lower input load.

(3) Study on the relationship between stress intensity factor, eccentricity and indenter width can enable us to optimize flat tooth cone design, reduce energy consumption and improve rock breaking efficiency.

\section{Acknowledgment}

Foundation item: Supported by the National Natural Science Foundation of China (11272141).

\section{References}

[1] H Mroueh, I Shahrour, "A simplified 3D model for tunnel construction using tunnel boring machines," Tunneling and Underground Space Technology, vol.23, no.1, pp.38-45, (2008)

[2] Y Koyama, "Present status and technology of shield tunneling method in Japan," Tunneling and Underground Space Technology, vol.18, no.2, pp.145-159, (2003)

[3] Lu Yang, Ma Jiming, Xu Qianjun, Han Moning, "TBM in the future of China, Marine Georesources and Geotechnology," vol.22, no.3, pp.185-193, (2004)http://xueshu.baidu.com/s?wd=author\%3A\%28QIANJUN\%20\%20\%20XU\%29\%20\&tn=SE _baiduxueshu_c1gjeupa\&ie=utf-8\&sc_f_para=sc_hilight $\% 3$ Dperson

[4] Wang Mengru, Li Dianhuang, Zhang Jingjian, "Rock tunnel boring machine (TBM) construction and engineering examples," China Railway Press, (2004)

[5] MJ Jackson, MP Hitchiner, "High Performance Grinding and Advanced Cutting Tools," Springer New York, (2013)

[6] AM Adaskin, VN Butrim, "Study of wear rate of cutting tools made from Cr-based high-temperature alloy during operation depending on the material properties of the cutting tool and the rate of cutting," Journal of Friction \& Wear, vol.35, no.5, pp.407-413, (2014)

[7] Richard Edward Gertsch, "Rock toughness and disc cutting," Ph.D. dissertation, University of Missouri Rolla, USA, pp.5, (2000)

[8] YV Rubtsov, GV Konnova, VS Shchetinin, SV Zolotoreva, "Improving the cutting mechanism of a disk-tupe wood chipper,” Russian Engineering Research, vol.31, no.1, pp.28-30, (2011)

[9] Zhang Zhao huang, Sun Fei, "The three-dimension model for the rock-breaking mechanism of disc cutter and analysis of rock-breaking forces," Acta Mechanica Sinica, vol.28, no.3, pp.675-682, (2012)

[10] Chen Yinghua, "Experimental study of rock-breaking with an offset single cone bit," Petroleum Science, vol.5, no.2, pp.179-182, (2008)

[11] Tan Qing, Zhang Gui-ju, "Differentiation and analysis on rock breaking characteristics of TBM disc cutter at different rock temperatures," Journal of Central South University, vol.22, no.12, pp.4807-4818, (2015)

[12] Choi Sung-Oong, Lee Seung-Joong, "Numerical study to estimate the cutting power on a disc cutter in jointed rock mass," Tunnel Engineering, vol.20, no.1, pp.440-451, (2016)

[13] Zhang Zhaohuang, Hu Xiumei, Meng Liang, Sun Fei, "Theoretical analysis of efficiency of rock breaking by disc cutters," Journal of Basic Science and Engineering, vol.S, no.1, pp.199-206, (2012) 
[14] Zhang Zhaohuang, Sun Fei, Meng Liang, "The comparative analysis of rocks' resistance to forward-slanting disc cutters and traditionally installed disc cutters,” Acta Mechanica Sinica, pp.1-6, (2015)

[15] V.Magnenet, C.Auvray, S.Djordem and F.Homand, "On the estimation of elastoplastic properties of rocks by indentation tests,” International Journal of Rock Mechanics and Mining Sciences, vol.46, no.3, pp.635-642, (2009)

[16] A.Carpinteri, B.Chiaia and S.Invernizzi, "Numerical analysis of indentation fracture in quasi-brittle materials," Engineering Fracture Mechanics, vol.71, no.4, pp.567-577, (2004)

[17] R.Mouginot and D.Maugis, "Fracture indentation beneath flat and spherical punches, Journal of materials science," vol.20, no.12, pp.4354-4376, (1985)

[18] L.Nicola, A.F.Bower, A.Needleman, "Surface versus bulk nucleation of dislocations during contact," Journal of the Mechanics and Physics of Solids, vol.55, no.6, pp.1120-1144, (2007)

[19] M.I.Porter, D.A.Hills, "Note on the complete contact between a flat rigid punch and an elastic layer attached to a dissimilar substrate," International Journal of Mechanical Sciences, vol.44, no.3, pp.509-520, (2002)

[20] A. E. Giannakopoulos, T. C. Lindley, and S. Suresh, "Aspects of equivalence between contact mechanics and fracture mechanics: Theoretical connections and a life-prediction methodology for fretting-fatigue," Acta Materialia, vol.46, no.9, pp.2599-2968, (1998)

[21] A. E. Giannakopoulos and S. Suresh, "Theory of indentation of piezoelectric materials," Acta Materialia, vol.47, no.7, pp.2153-2164, (1999)

[22] A. C. Fischer-Cripps, "Predicting Hertzian fracture, Journal of Materials Science," vol.32, no.5, pp.1277-1285, (1997)

[23] B. Yang and S. Mall, “On crack initiation mechanism in fretting fatigue," Journal of Applied Mechanics, vol.68, no.1, pp.76-80, (2001)

[24] Martin Entacher, E. Schuller, R. Galler, "Rock failure and crack propagation beneath disc cutters," Rock Mechanics \& Rock Engineering, vol.48, no.4, pp.1559-1572, (2015)

[25] V. Buljak, G. Cocchetti, G. Maier, "Calibration of brittle fracture models by sharp indenters and inverse analysis," International Journal of Fracture, vol.184, no.1-2, pp.231-235, (2013)

[26] Nadai A., Hodge P. G., "Theory of flow and fracture of solids," Ph.D. dissertation, Harvard University, USA, (1963)

[27] Xie Yujun, Hills D.A, "Quasibrittle fracture beneath a flat bearing surface," Engineering Fracture Mechanics, vol.75, no.5, pp.1223-1230, (2008)

[28] Xie Yujun, Wang Xiaohua, Hu Xiaozhi, Zhu Xiangzhe, "Fracture-based model of periodic-arrayed indentation for rock cutting," International Journal of Rock Mechanics \& Mining Sciences, vol.76, pp.217-221, (2014)

[29] Xie Yujun, Lee K.Y., Hu Xiaozhi, Cai Yongmei, "Applications of conservation integral to indentation with a rigid punch,” Engineering Fracture Mechanics, vol.76, no.7, pp.949-957, (2009)

[30] Budiansky B, Rice JR, “Conservation Laws and Energy-Release Rates,” Journal of Applied Mechanics, vol.40, no.1, pp.201-203, (1973)

[31] Tada H, Paris PC, Irwin GR, "The stress analysis of cracks handbook," ASME Press, (2000)

[32] Rice JR, "A path-independent integral and the approximate analysis of strain concentrated by notches and cracks,” ASME J Appl Mech, vol.35, no.2, pp.379-386, (1968)

[33] Xie Yujun, Li Peining, Xu H, "On KI estimates of cracked pipes using an elliptical hole model and elementary beam strength theory of cracked beams,” Engineering Fracture Mechanics, vol.59, no.3, pp.299-402, (1998)

[34] Xie Yujun, Zhang X, Wang Xiaohua, "An exact method on penny-shape crack homogeneous and composite cylinders,” International Journal of Solids \& Structures, vol.38, no.38-39, pp.6953-6963, (2001)

[35] Xie Yujun, "An analytical method on circumferential periodic cracked pipes and shells," International Journal of Solids \& Structures, vol.37, no.37, pp.5189-5201, (2000) 


\section{Authors}

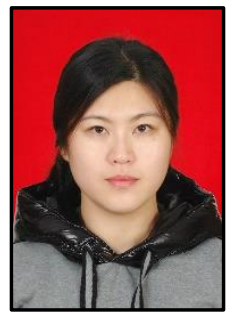

Biography: Yili Duo, female, Ph.D. candidate, research direction: fracture mechanics. E-mail: lnpuduoyili@163.com 